Proceedings of the Edinburgh Mathematical Society (2003) 46, 597-607 (C)

DOI:10.1017/S0013091502000779 Printed in the United Kingdom

\title{
MULTIPLE POSITIVE SOLUTIONS AND BIFURCATION FOR AN EQUATION RELATED TO CHOQUARD'S EQUATION
}

\author{
TASSILO KÜPPER ${ }^{1}$, ZHENGJIE ZHANG ${ }^{2}$ AND HONGQIANG XIA ${ }^{3}$ \\ ${ }^{1}$ Mathematisches der Universität zu Köln, Albertus-Magnus Platz, \\ 50923 Köln, Germany \\ ${ }^{2}$ Department of Mathematics, Central China Normal University, \\ Wuhan 430079, People's Republic of China (zhang@sissa.it) \\ ${ }^{3}$ Department of Mathematics, University of Manchester, \\ Oxford Road, Manchester M13 9PL, UK
}

(Received 13 August 2002)

Abstract In this paper we study the existence of multiple positive solutions and the bifurcation problem for the following equation:

$$
-\Delta u+u=\left(\int_{\mathbb{R}^{3}} \frac{|u(y)|^{2}}{|x-y|} \mathrm{d} y\right) u+\mu f(x), \quad x \in \mathbb{R}^{3},
$$

where $f(x) \in H^{-1}\left(\mathbb{R}^{3}\right), f(x) \geqslant 0, f(x) \not \equiv 0$. We show that there are positive constants $\mu^{*}$ and $\mu^{* *}$ such that the above equation possesses at least two positive solutions for $\mu \in\left(0, \mu^{*}\right)$, and no positive solution for $\mu>\mu^{* *}$. Furthermore, we prove that $\mu=\mu^{*}$ is a bifurcation point for the equation under study.

Keywords: multiple solutions; Choquard's equation; bifurcation

2000 Mathematics subject classification: Primary 35J60; 35J70

\section{Introduction and main results}

The study of inhomogeneous non-local problems has been a very active topic in recent years and many interesting results have been obtained by different authors (see, for example, $[4-\mathbf{6}, \mathbf{8}, \mathbf{1 0}, \mathbf{1 1}, \mathbf{1 6}])$. But most of the authors cited have not discussed the properties of the solutions, rather they only investigated the existence of the solutions. In this paper we consider the equation

$$
-\Delta u+u=\left(\int_{\mathbb{R}^{3}} \frac{|u(y)|^{2}}{|x-y|} \mathrm{d} y\right) u+\mu f(x), \quad x \in \mathbb{R}^{3},
$$

where $\mu \geqslant 0$ is a given constant, and $f(x)$ satisfies the conditions

$$
\left.\begin{array}{c}
f(x) \in H^{-1}\left(\mathbb{R}^{3}\right), \\
f(x) \geqslant 0, \quad f(x) \not \equiv 0, \quad \forall x \in \mathbb{R}^{3},
\end{array}\right\}
$$


which can be regarded as a perturbation of the homogeneous equation

$$
-\Delta u+u=\left(\int_{\mathbb{R}^{3}} \frac{|u(y)|^{2}}{|x-y|} \mathrm{d} y\right) u, \quad x \in \mathbb{R}^{3} .
$$

We not only study the existence of multiple positive solutions of the above problem but also investigate the properties of the solutions. In particular we study the dependence of solutions on the parameter $\mu$, and work out the bifurcation point of problem (1.1).

The main idea of our paper is related to the bifurcation problems for semilinear elliptic equations $[\mathbf{7}, \mathbf{9}, \mathbf{1 2}]$. The methods used in these papers are strongly dependent on the local character of the equation. We use a different method and some special estimates to obtain our results for the non-homogeneous Choquard's equation. The main results of this paper are as follows.

Theorem 1.1. Suppose $f(x)$ satisfies $(A)$, then there are positive constants $\mu^{*}$ and $\mu^{* *}$ such that

(1) Equation (1.1) possesses at least two positive solutions for all $\mu \in\left(0, \mu^{*}\right)$;

(2) there is no positive solution of Equation (1.1) for all $\mu>\mu^{* *}$.

Theorem 1.2. Under the condition of Theorem 1.1, there is only one positive solution for Equation (1.1) for $\mu=\mu^{*}$, and $\mu=\mu^{*}$ is a bifurcation point for Equation (1.1).

The paper is organized as follows. In $\S 2$ we introduce some notation and facts we need later. In $\S 3$ we prove Theorem 1.1 stated in $\S 1$. In $\S 4$ we discuss the properties and bifurcation of solutions of Equation (1.1) and prove Theorem 1.2.

\section{Some notation and preliminary results}

In this section we give some notation, definitions and state several preliminary results that will be used in the subsequent sections. Let

$$
\langle u, v\rangle=\int_{\mathbb{R}^{3}}(\nabla u \nabla v+u v) \mathrm{d} x
$$

denote the inner product of $H^{1}\left(\mathbb{R}^{3}\right)$, and let

$$
\|u\|=\langle u, u\rangle^{1 / 2}
$$

be the norm in the Hilbert space $H^{1}\left(\mathbb{R}^{3}\right)$, whose dual space $\left(H^{1}\left(\mathbb{R}^{3}\right)\right)^{*}=H^{-1}\left(\mathbb{R}^{3}\right)$ has norm denoted by $\|\cdot\|_{H^{-1}}$. We say that $u \in H^{1}\left(\mathbb{R}^{3}\right)$ is a weak solution of Equation (1.1) if

$$
\int_{\mathbb{R}^{3}}(\nabla u \nabla v+u v) \mathrm{d} x=\int_{\mathbb{R}^{3}}\left(\int_{\mathbb{R}^{3}} \frac{u^{2}(y)}{|x-y|} \mathrm{d} y\right) u v \mathrm{~d} x-\mu \int_{\mathbb{R}^{3}} f v \mathrm{~d} x, \quad \forall v \in H^{1}\left(\mathbb{R}^{3}\right) .
$$

One can verify that the weak solutions of (1.1) are equivalent to the non-zero critical points of the functional

$$
I_{\mu, f}(u)=\frac{1}{2}\|u\|^{2}-\frac{1}{4} \iint_{\mathbb{R}^{3} \times \mathbb{R}^{3}} \frac{u^{2}(x) u^{2}(y)}{|x-y|} \mathrm{d} x \mathrm{~d} y-\mu \int_{\mathbb{R}^{3}} f(x) u(x) \mathrm{d} x .
$$


Define

$$
I(u)=\frac{1}{2}\|u\|^{2}-\frac{1}{4} \iint_{\mathbb{R}^{3} \times \mathbb{R}^{3}} \frac{u^{2}(x) u^{2}(y)}{|x-y|} \mathrm{d} x \mathrm{~d} y .
$$

Denote

$$
I^{\infty}=\inf \left\{I(u) \mid u \in H^{1}\left(\mathbb{R}^{3}\right), u \geqslant 0, u \neq 0, F(u)=1\right\},
$$

where $F(u)$ is the functional defined in $H^{1}\left(\mathbb{R}^{3}\right)$, by

$$
F(u)= \begin{cases}0 & \text { if } u \equiv 0, \\ \frac{\int_{\mathbb{R}^{3}}\left(|\nabla u|^{2}+|u|^{2}\right) \mathrm{d} x}{\iint_{\mathbb{R}^{3} \times \mathbb{R}^{3}} \frac{u(x)^{2} u(y)^{2}}{|x-y|} \mathrm{d} x \mathrm{~d} y} & \text { if } u \neq 0 .\end{cases}
$$

From $[4,10]$, we know that $I^{\infty}$ is achieved by a function $\omega \in H^{1}\left(\mathbb{R}^{3}\right)$, which is a solution of Equation (1.2).

For a $C^{1}$-functional $J$ on a Banach space $E, C \in \mathbb{R}^{1}$, we call $\left\{u_{n}\right\}$ a $(\mathrm{PS})_{C}$ sequence of $J$ on $E$ if

$$
J\left(u_{n}\right) \rightarrow C \text { and } \quad J^{\prime}\left(u_{n}\right) \rightarrow 0 .
$$

In order to prove our results, we need four lemmas as follows.

Lemma 2.1. $I_{\mu, f}(u) \in C^{1}\left(H^{1}\left(\mathbb{R}^{3}\right), \mathbb{R}\right)$ and

$$
\left\langle I_{\mu, f}^{\prime}(u), h\right\rangle=\langle u, h\rangle-\iint_{\mathbb{R}^{3} \times \mathbb{R}^{3}} \frac{u^{2}(y) u(x) h(x)}{|x-y|} \mathrm{d} y \mathrm{~d} x-\mu \int_{\mathbb{R}^{3}} f(x) h(x) \mathrm{d} x .
$$

Lemma 2.1 can be proved in a standard way, therefore we omit the proof here.

Lemma 2.2. Let $\left(P_{n}\right)_{n>1}$ be a sequence in $L^{1}\left(\mathbb{R}^{3}\right)$ satisfying $P_{n} \geqslant 0$ in $\mathbb{R}^{3}$ and $\int_{\mathbb{R}^{3}} P_{n} \mathrm{~d} x=L$, where $L>0$ is fixed. Then there exists a subsequence $\left(P_{n_{k}}\right)_{k>1}$ satisfying one of the following three possibilities.

(I) (compactness). There exists $y_{k} \in \mathbb{R}^{3}$ such that $P_{n_{k}}\left(x+y_{k}\right)$ is tight, i.e.

$$
\forall \epsilon>0, \quad \exists R<\infty \int_{y_{k}+B_{R}} P_{n_{k}}(x) \mathrm{d} x \geqslant L-\epsilon .
$$

(II) (vanishing).

$$
\lim _{k \rightarrow \infty} \sup _{y \in \mathbb{R}^{3}} \int_{y+B_{R}} P_{n_{k}}(x) \mathrm{d} x=0 \quad \text { for all } R<\infty
$$

(III) (dichotomy). There exists $\alpha \in(0, L)$ such that for any $\epsilon \geqslant 0$ there exists $k_{0}>1$ and $P_{k}^{1}, P_{k}^{2} \in L_{+}^{1}\left(\mathbb{R}^{3}\right)$ satisfying, for $k \geqslant k_{0}$,

$$
\begin{gathered}
\operatorname{dist}\left(\operatorname{supp}\left\{P_{k}^{1}\right\}, \operatorname{supp}\left\{P_{k}^{2}\right\}\right) \rightarrow \infty, \\
\left\|P_{n_{k}}-\left(P_{k}^{1}+P_{k}^{2}\right)\right\|_{L^{1}\left(\mathbb{R}^{3}\right)} \leqslant \epsilon, \\
\left|\int_{\mathbb{R}^{3}} P_{k}^{1} \mathrm{~d} x-\alpha\right| \leqslant \epsilon, \quad\left|\int_{\mathbb{R}^{3}} P_{k}^{2} \mathrm{~d} x-(L-\alpha)\right| \leqslant \epsilon .
\end{gathered}
$$


Lemma 2.2 can be found in $[\mathbf{1 3}, \mathbf{1 4}]$ and the following Lemmas 2.3 and 2.4 are cited from $[6]$.

Lemma 2.3. Suppose $\left\{u_{n}\right\}$ is bounded in $H^{1}\left(\mathbb{R}^{3}\right), u_{0} \in H^{1}\left(\mathbb{R}^{3}\right)$ and $u_{n} \rightarrow u_{0}$ (weakly in $\left.H^{1}\left(\mathbb{R}^{3}\right)\right)$. Then we have

$$
\lim _{n \rightarrow \infty} \iint_{\mathbb{R}^{3} \times \mathbb{R}^{3}} \frac{u_{n}^{2}(x) u_{n}^{2}(y)-u_{0}^{2}(x) u_{0}^{2}(y)-\left(u_{n}-u_{0}\right)^{2}(x)\left(u_{n}-u_{0}\right)^{2}(y)}{|x-y|} \mathrm{d} x \mathrm{~d} y=0 .
$$

Lemma 2.4. Suppose $\left\{u_{n}\right\} \in H^{1}\left(\mathbb{R}^{3}\right)$ is a $(\mathrm{PS})_{C}$ sequence of $I_{\mu, f}$ and $u_{0} \in H^{1}\left(\mathbb{R}^{3}\right)$, $u_{n} \rightarrow u_{0}$ (weakly in $H^{1}\left(\mathbb{R}^{3}\right)$ ). Then $I_{\mu, f}^{\prime}\left(u_{0}\right)=0$, i.e. $u_{0}$ is a weak solution of (1.1).

\section{Existence and non-existence results}

In this section, we shall prove Theorem 1.1. First we define

$$
\bar{B}_{R}=\left\{u \in H^{1}\left(\mathbb{R}^{3}\right) \mid\|u\| \leqslant R\right\}
$$

and

$$
I_{0}=I_{0}(R)=\inf _{u \in \bar{B}_{R}} I_{\mu, f}(u)
$$

Theorem 3.1. Suppose $\left\{u_{n}\right\}$ is a (PS) $C$ sequence of $I_{\mu, f}(u)$ in $H^{1}\left(\mathbb{R}^{3}\right)$ and $u_{0} \in$ $H^{1}\left(\mathbb{R}^{3}\right), u_{n} \rightarrow u_{0}\left(\right.$ weakly in $\left.H^{1}\left(\mathbb{R}^{3}\right)\right)$. Then either $u_{n} \rightarrow u_{0}\left(\right.$ strongly in $\left.H^{1}\left(\mathbb{R}^{3}\right)\right)$ and $I_{\mu, f}\left(u_{0}\right)=C$, or $C \geqslant I_{\mu, f}\left(u_{0}\right)+I^{\infty}$.

Proof. Since $\left\{u_{n}\right\}$ is a $(\mathrm{PS})_{C}$ sequence of $I_{\mu, f}$ in $H^{1}\left(\mathbb{R}^{3}\right)$, then

$$
\begin{aligned}
& I_{\mu, f}\left(u_{n}\right)=C+o(1), \\
& \left\langle I_{\mu, f}^{\prime}\left(u_{n}\right), u_{n}\right\rangle=o(1) .
\end{aligned}
$$

From $u_{n} \rightarrow u_{0}$ (weakly in $H^{1}\left(\mathbb{R}^{3}\right)$ ), we get for $v_{n}=u_{n}-u_{0}$

$$
\begin{array}{ll}
v_{n} \rightarrow 0 & \text { weakly in } H^{1}\left(\mathbb{R}^{3}\right), \\
v_{n} \rightarrow 0 & \text { a.e. on } \mathbb{R}^{3} .
\end{array}
$$

From Lemma 2.3 and Lemma 2.4 we obtain

$$
C+o(1)=I_{\mu, f}\left(u_{n}\right)=I_{\mu, f}\left(u_{0}\right)+I\left(v_{n}\right)+o(1)
$$

and

$$
\begin{aligned}
o(1) & =\left\langle I_{\mu, f}^{\prime}\left(u_{n}\right), u_{n}\right\rangle \\
& =\left\langle I_{\mu, f}^{\prime}\left(u_{0}\right), u_{0}\right\rangle+\left\langle I_{\mu, f}^{\prime}\left(v_{n}\right), v_{n}\right\rangle+o(1) \\
& =\left\langle I^{\prime}\left(v_{n}\right), v_{n}\right\rangle+o(1) .
\end{aligned}
$$

If

$$
v_{n} \rightarrow 0 \quad\left(\text { strongly in } H^{1}\left(\mathbb{R}^{3}\right)\right),
$$


then

$$
\left.u_{n} \rightarrow u_{0} \quad \text { (strongly in } H^{1}\left(\mathbb{R}^{3}\right)\right)
$$

and

$$
I_{\mu, f}\left(u_{0}\right)=\lim _{n \rightarrow \infty} I_{\mu, f}\left(u_{n}\right)=C .
$$

If $v_{n}$ does not strongly converge to zero in $H^{1}\left(\mathbb{R}^{3}\right)$, we may assume that

$$
\left\|v_{n}\right\| \rightarrow \eta>0 \text {. }
$$

Then from (3.5) and (3.6) we can get

$$
\begin{aligned}
C= & I_{\mu, f}\left(u_{0}\right)+I\left(v_{n}\right)+o(1), \\
& \left\langle I^{\prime}\left(v_{n}\right), v_{n}\right\rangle=o(1) .
\end{aligned}
$$

Using the same method as in $[\mathbf{1}],[\mathbf{2}]$ and $[\mathbf{3}]$ we can prove that

$$
I\left(v_{n}\right) \geqslant I^{\infty}+o(1) .
$$

Then we have

$$
C=I_{\mu, f}\left(u_{0}\right)+I\left(v_{n}\right)+o(1) \geqslant I_{\mu, f}\left(u_{0}\right)+I^{\infty} .
$$

Theorem 3.2. Under condition $A$ on $f(x)$, there are constants $\mu, R$ and $u_{0, \mu} \in$ $H^{1}\left(\mathbb{R}^{3}\right)$ such that $I_{0}(R)=I_{\mu, f}\left(u_{0, \mu}\right), I_{\mu, f}^{\prime}\left(u_{0, \mu}\right)=0$, i.e. $u_{0, \mu}$ is a positive solution of Equation (1.1).

Proof. For any $u \in H^{1}\left(\mathbb{R}^{3}\right)$ and $u>0$, we have

$$
\begin{aligned}
I_{\mu, f}(t u) & =\frac{t^{2}}{2} \int_{\mathbb{R}^{3}}\left(|\nabla u|^{2}+u^{2}\right) \mathrm{d} x-\frac{t^{4}}{4} \iint_{\mathbb{R}^{3} \times \mathbb{R}^{3}} \frac{u^{2}(x) u^{2}(y)}{|x-y|} \mathrm{d} x \mathrm{~d} y-t \mu \int_{\mathbb{R}^{3}} f(x) u \mathrm{~d} x, \\
\frac{\mathrm{d} I_{\mu, f}(t u)}{\mathrm{d} t} & =t \int_{\mathbb{R}^{3}}\left(|\nabla u|^{2}+u^{2}\right) \mathrm{d} x-t^{3} \iint_{\mathbb{R}^{3} \times \mathbb{R}^{3}} \frac{u^{2}(x) u^{2}(y)}{|x-y|} \mathrm{d} x \mathrm{~d} y-\mu \int_{\mathbb{R}^{3}} f(x) u \mathrm{~d} x .
\end{aligned}
$$

From (3.8) we know that there is a constant $\bar{t}$ such that

$$
\frac{\mathrm{d} I_{\mu, f}(t u)}{\mathrm{d} t}<0
$$

for $t<\bar{t}$. Hence $I_{\mu, f}(t u)$ is a decreasing function in $t \in(0, \bar{t})$.

On the other hand, we have

$$
\int_{\mathbb{R}^{3}} f(x) u \mathrm{~d} x \leqslant\|f\|_{H^{-1}} \times\|u\|_{H^{1}}
$$


and

$$
\begin{aligned}
\iint_{\mathbb{R}^{3} \times \mathbb{R}^{3}} \frac{u^{2}(x) u^{2}(y)}{|x-y|} \mathrm{d} x \mathrm{~d} y & +\iint_{\mathbb{R}^{3} \times \mathbb{R}^{3} \cap\{|x-y|<\delta\}}\left(\frac{u^{2}(x) u^{2}(y)}{|x-y|}\right) \mathrm{d} x \mathrm{~d} y \\
= & \iint_{\mathbb{R}^{3} \times \mathbb{R}^{3} \cap\{|x-y| \geqslant \delta\}}+\frac{1}{\delta}\left(\int_{\mathbb{R}^{3}} u^{2}(x) \mathrm{d} x\right)^{2}+C \delta\left(\int_{\mathbb{R}^{3}} u^{2}(x) \mathrm{d} x\right)\left(\int_{\mathbb{R}^{3}}|\nabla u|^{2} \mathrm{~d} x\right),
\end{aligned}
$$

where we have used the Hölder and Sobolev inequalities; see [13], [14] and [12] for more details.

From (3.8)-(3.10) we know that for $\mu$ sufficiently small $I_{\mu, f}(t u)$ is an increasing function for $t \in\left(\bar{t}, t_{1}\right)$. This shows that for any $u>0$ and $u \in H^{1}\left(\mathbb{R}^{3}\right), I_{\mu, f}(t u)$ achieves a local minimum at $t=\bar{t}$. Since $I_{\mu, f}(0)=0$, there is an $R>0$ such that $I_{0}=I_{0}(R)=$ $\inf _{u \in B_{R}} I_{\mu, f}(u)<0$.

Using Ekeland's variational principle [8], for $R$ small, we obtain a $(\mathrm{PS})_{C}$ sequence of $I_{\mu, f}\left(u_{n}\right)$. This means that we obtain a sequence $u_{n} \in H^{1}\left(\mathbb{R}^{3}\right)$ such that

$$
\begin{aligned}
& I_{\mu, f}\left(u_{n}\right)=I_{0}(R)+o(1), \\
& I_{\mu, f}^{\prime}\left(u_{n}\right) \rightarrow 0 .
\end{aligned}
$$

Since $I_{0}(R)<0$, from Theorem 3.1, we know that there is an $u_{0, \mu} \in H^{1}\left(\mathbb{R}^{3}\right)$ such that

$$
u_{n} \rightarrow u_{0, \mu} \quad \text { strongly in } H^{1}\left(\mathbb{R}^{3}\right) .
$$

Thus,

$$
\begin{gathered}
I_{0}(R)=I_{\mu, f}\left(u_{0, \mu}\right), \\
I_{\mu, f}^{\prime}\left(u_{0, \mu}\right)=0 .
\end{gathered}
$$

This shows that $u_{0, \mu}$ is a solution of Equation (1.1).

Let $u_{0, \mu}$ be the function given in Theorem 3.2 , and $\omega$ the function achieving the infimum in (2.3), then under the condition of Theorem 3.2 we have

$$
\frac{\mathrm{d} I_{\mu, f}(t \omega)}{\mathrm{d} t}>0 \text { for all } t \in\left(\bar{t}, t_{1}\right)
$$

We define

$$
\Gamma=\left\{\gamma \in C^{1}\left([0,1], H^{1}\left(\mathbb{R}^{3}\right)\right): \gamma(0)=u_{0, \mu}, \gamma(1)=u_{0, \mu}+t_{1} \omega\right\}
$$

and

$$
\bar{C}=\inf _{\gamma \in \Gamma} \sup _{u \in \gamma} I_{\mu, f}(u) .
$$

Theorem 3.3. $\bar{C}<I_{0}+I^{\infty}$, where $I_{0}=\inf _{u \in \bar{B}_{R_{0}}} I_{\mu, f}(u)$ for $R_{0}>0$ small enough, and $I^{\infty}=I(\omega)$. 
Proof. If

$$
\sup _{t>0} I_{\mu, f}\left(u_{0, \mu}+t \omega\right)<I_{0}+I^{\infty},
$$

then by the definition of $\bar{C}$ we have

$$
\bar{C}<I_{0}+I^{\infty} .
$$

Since $u_{0, \mu}$ and $\omega$ are positive functions and $t>0$, we have

$$
\begin{aligned}
I_{\mu, f}\left(u_{0, \mu}+t \omega\right)=\frac{1}{2} & \int_{\mathbb{R}^{3}}\left(\left|\nabla\left(u_{0, \mu}+t \omega\right)\right|^{2}+\left(u_{0, \mu}+t \omega\right)^{2}\right) \mathrm{d} x-\mu \int_{\mathbb{R}^{3}} f\left(u_{0, \mu}+t \omega\right) \mathrm{d} x \\
& -\frac{1}{4} \iint_{\mathbb{R}^{3} \times \mathbb{R}^{3}} \frac{\left(u_{0, \mu}+t \omega\right)^{2}(x)\left(u_{0, \mu}+t \omega\right)^{2}(y)}{|x-y|} \mathrm{d} x \mathrm{~d} y \\
< & \frac{1}{2} \int_{\mathbb{R}^{3}}\left(\left|\nabla u_{0, \mu}\right|^{2}+u_{0, \mu}^{2}\right) \mathrm{d} x+\frac{t^{2}}{2} \int_{\mathbb{R}^{3}}\left(|\nabla \omega|^{2}+\omega^{2}\right) \mathrm{d} x \\
& +t \int_{\mathbb{R}^{3}}\left(\nabla u_{0, \mu} \nabla \omega+u_{0, \mu} \omega\right) \mathrm{d} x-\frac{1}{4} \iint_{\mathbb{R}^{3} \times \mathbb{R}^{3}} \frac{u_{0, \mu}^{2}(x) u_{0, \mu}^{2}(y)}{|x-y|} \mathrm{d} x \mathrm{~d} y \\
& -\frac{2 t}{4} \iint_{\mathbb{R}^{3} \times \mathbb{R}^{3}} \frac{u_{0, \mu}^{2}(x) u_{0, \mu}(y) \omega(y)+u_{0, \mu}^{2}(y) u_{0, \mu}(x) \omega(x)}{|x-y|} \mathrm{d} x \mathrm{~d} y \\
& -\frac{t^{4}}{4} \iint_{\mathbb{R}^{3} \times \mathbb{R}^{3}} \frac{\omega^{2}(x) \omega^{2}(y)}{|x-y|} \mathrm{d} x \mathrm{~d} y-\mu \int_{\mathbb{R}^{3}} f u_{0, \mu} \mathrm{d} x-\mu t \int_{\mathbb{R}^{3}} f \omega \mathrm{d} x .
\end{aligned}
$$

Since $u_{0, \mu}$ is a solution of (1.1), we have

$$
\int_{\mathbb{R}^{3}}\left(\nabla u_{0, \mu} \nabla \omega+u_{0, \mu} \omega\right) \mathrm{d} x=\iint_{\mathbb{R}^{3} \times \mathbb{R}^{3}} \frac{u_{0, \mu}^{2}(y) u_{0, \mu}(x) \omega(x)}{|x-y|} \mathrm{d} x \mathrm{~d} y+\mu t \int_{\mathbb{R}^{3}} f \omega \mathrm{d} x .
$$

From (3.13) and (3.14), we get

$$
\sup _{t>0} I_{\mu, f}\left(u_{0, \mu}+t \omega\right)<I_{0}+I^{\infty} .
$$

Then

$$
\bar{C}<I_{0}+I^{\infty}
$$

Theorem 3.4. Under the condition of Theorem 3.2, there are at least two positive solutions of Equation (1.1).

Proof. First, from Theorem 3.2, we obtain a solution $u_{0, \mu}$ of Equation (1.1).

Using the Mountain Pass Lemma and Theorem 3.3, we can get a sequence $\left\{u_{n}\right\}$, $u_{n} \in H^{1}\left(\mathbb{R}^{3}\right)$, which is a $(\mathrm{PS})_{\bar{C}}$ sequence of $I_{\mu, f}$. It is easy to prove that $u_{n}$ is bounded in $H^{1}\left(\mathbb{R}^{3}\right)$. Hence there is a subsequence $u_{n_{k}}$ and a $u_{1, \mu} \in H^{1}\left(\mathbb{R}^{3}\right)$ such that

$$
u_{n_{k}} \rightarrow u_{1, \mu} \quad \text { weakly in } H^{1}\left(\mathbb{R}^{3}\right) .
$$


Using Theorem 3.1, we know that $u_{n_{k}} \rightarrow u_{1, \mu}$ is strongly convergent in $H^{1}\left(\mathbb{R}^{3}\right)$, hence $u_{1, \mu}$ is a solution of Equation (1.1).

By the strong maximum principle for elliptic equations and Theorems 3.2 and 3.3, we know that $u_{0, \mu}$ and $u_{1, \mu}$ are two different positive solutions of Equation (1.1).

Theorem 3.5. Under the condition (A) on $f(x)$, there is a positive constant $\bar{\mu}$ such that Equation (1.1) has no positive solution whenever $\mu>\bar{\mu}$.

Proof. In fact, for any $u>0$ we have

$$
\begin{aligned}
& \iint_{\mathbb{R}^{3} \times \mathbb{R}^{3}} \frac{\omega^{2}(y) \omega(x) u(x)}{|x-y|} \mathrm{d} x \mathrm{~d} y \\
& \leqslant \iint_{\mathbb{R}^{3} \times \mathbb{R}^{3}} \frac{\omega^{2}(y) \omega(x)^{2}}{|x-y|} \mathrm{d} x \mathrm{~d} y+\iint_{\mathbb{R}^{3} \times \mathbb{R}^{3}} \frac{u^{2}(y) \omega^{2}(x)}{|x-y|} \mathrm{d} x \mathrm{~d} y,
\end{aligned}
$$

where $\omega$ is a positive solution of Equation (1.2) (this can be obtained from Lemma 3.1 in $[\mathbf{2}])$. If Equation (1.1) possesses a positive solution, say $u$, then

$$
\int_{\mathbb{R}^{3}}(-\Delta u+u) v \mathrm{~d} x=\iint_{\mathbb{R}^{3} \times \mathbb{R}^{3}} \frac{u^{2}(y) u(x) v(x)}{|x-y|} \mathrm{d} x \mathrm{~d} y+\mu \int_{\mathbb{R}^{3}} f v \mathrm{~d} x
$$

for any $v \in H_{0}^{1}\left(\mathbb{R}^{3}\right)$.

Taking $v=\omega$ in (3.16) and using (3.15), we can get

$$
\mu \int_{\mathbb{R}^{3}} f \omega \mathrm{d} x \leqslant \iint_{\mathbb{R}^{3} \times \mathbb{R}^{3}} \frac{\omega^{2}(x) \omega^{2}(y)}{|x-y|} \mathrm{d} x \mathrm{~d} y .
$$

Set

$$
\bar{\mu}=\frac{\iint_{\mathbb{R}^{3} \times \mathbb{R}^{3}} \frac{\omega^{2}(x) \omega^{2}(y)}{|x-y|} \mathrm{d} x \mathrm{~d} y}{\int_{\mathbb{R}^{3}} f(x) \omega(x) \mathrm{d} x} .
$$

From (3.16) and (3.17), we know that if Equation (1.1) possesses a positive solution, then $\mu \leqslant \bar{\mu}$.

We are now in a position to prove Theorem 1.1.

Proof of Theorem 1.1. From Theorem 3.5 we know there is no positive solution for Equation (1.1) if $\mu>\bar{\mu}$. So we take

$$
\mu^{* *}=\inf \left\{\mu \in \mathbb{R}^{+} \mid \text {there is no positive solution for Equation (1.1) }\right\} .
$$

From Theorems 3.2 and 3.3 we know that, if $\mu$ is small enough, there are at least two positive solutions for Equation (1.1). So set

$$
\mu^{*}=\sup \left\{\mu \in \mathbb{R}^{+} \mid \text {there are at least two positive solutions for Equation (1.1) }\right\} .
$$

This completes the proof of Theorem 1.1. 


\section{Properties and bifurcation of solutions}

In this section we shall give some properties and study the bifurcation of solutions for Equation (1.1) and prove Theorem 1.2 stated in the introduction.

Proposition 4.1. The solution $u_{0, \mu}$ of Equation (1.1) converges strongly to 0 as $\mu \rightarrow 0$. That is

$$
\lim _{\mu \rightarrow 0}\left\|u_{0, \mu}\right\|_{H^{1}\left(\mathbb{R}^{3}\right)}=0 .
$$

Proof. From the previous section we know that

$$
I_{0}=I_{\mu, f}\left(u_{0, \mu}\right)=\inf _{u \in B_{r}} I_{\mu, f}(u)<0 .
$$

On the other hand, we have

$I_{\mu, f}\left(u_{0, \mu}\right)=\frac{1}{2} \int_{\mathbb{R}^{3}}\left(\left|\nabla u_{0, \mu}\right|^{2}+u_{0, \mu}^{2}\right) \mathrm{d} x-\frac{1}{4} \iint_{\mathbb{R}^{3} \times \mathbb{R}^{3}} \frac{u_{0, \mu}^{2}(x) u_{0, \mu}^{2}(y)}{|x-y|} \mathrm{d} x \mathrm{~d} y-\mu \int_{\mathbb{R}^{3}} f u_{0, \mu} \mathrm{d} x$

and

$$
\int_{\mathbb{R}^{3}}\left(\left|\nabla u_{0, \mu}\right|^{2}+u_{0, \mu}^{2}\right) \mathrm{d} x=\iint_{\mathbb{R}^{3} \times \mathbb{R}^{3}} \frac{u_{0, \mu}^{2}(x) u_{0, \mu}^{2}(y)}{|x-y|} \mathrm{d} x \mathrm{~d} y+\mu \int_{\mathbb{R}^{3}} f u_{0, \mu} \mathrm{d} x .
$$

So we get

$$
\begin{aligned}
I_{\mu, f}\left(u_{0, \mu}\right) & =\frac{1}{4} \int_{\mathbb{R}^{3}}\left(\left|\nabla u_{0, \mu}\right|^{2}+u_{0, \mu}^{2}\right) \mathrm{d} x-\frac{5}{4} \mu \int_{\mathbb{R}^{3}} f u_{0, \mu} \mathrm{d} x \\
& \geqslant \frac{1}{4}\left\|u_{0, \mu}\right\|^{2}-\frac{5}{4} \mu\|f\| \times\left\|u_{0, \mu}\right\|
\end{aligned}
$$

and

$$
\frac{1}{4}\left\|u_{0, \mu}\right\|^{2}-\frac{5}{4} \mu\|f\| \times\left\|u_{0, \mu}\right\| \leqslant 0 .
$$

Since $\left\|u_{0, \mu}\right\| \geqslant 0$, we get

$$
\left\|u_{0, \mu}\right\| \leqslant 5 \mu\|f\|
$$

Hence,

$$
\lim _{\mu \rightarrow 0}\left\|u_{0, \mu}\right\|=0
$$

Proposition 4.2. The solution $u_{1, \mu}$ of Equation (1.1) converges strongly to a non-zero solution of Equation (1.2) in $H^{1}\left(\mathbb{R}^{3}\right)$ as $\mu \rightarrow 0$.

Proof. From the previous section we know that

$$
I_{\mu, f}\left(u_{1, \mu}\right)=\inf _{\gamma \in \Gamma} \sup _{u \in \gamma} I_{\mu, f}(u),
$$

so

$$
I_{\mu, f}\left(u_{1, \mu}\right) \leqslant I^{\infty}
$$


That is,

$$
\frac{1}{2} \int_{\mathbb{R}^{3}}\left(\left|\nabla u_{1, \mu}\right|^{2}+u_{1, \mu}^{2}\right) \mathrm{d} x-\frac{1}{4} \iint_{\mathbb{R}^{3} \times \mathbb{R}^{3}} \frac{u_{1, \mu}^{2}(x) u_{1, \mu}^{2}(y)}{|x-y|} \mathrm{d} x \mathrm{~d} y-\mu \int_{\mathbb{R}^{3}} f u_{1, \mu} \mathrm{d} x \leqslant I^{\infty} .
$$

On the other hand, $u_{1, \mu}$ is a solution of Equation (1.1). Hence

$$
\int_{\mathbb{R}^{3}}\left(\left|\nabla u_{1, \mu}\right|^{2}+u_{1, \mu}^{2}\right) \mathrm{d} x=\iint_{\mathbb{R}^{3} \times \mathbb{R}^{3}} \frac{u_{1, \mu}^{2}(x) u_{1, \mu}^{2}(y)}{|x-y|} \mathrm{d} x \mathrm{~d} y+\mu \int_{\mathbb{R}^{3}} f u_{1, \mu} \mathrm{d} x .
$$

Combining (4.1) and (4.2), we get

$$
\frac{1}{4} \int_{\mathbb{R}^{3}}\left(\left|\nabla u_{1, \mu}\right|^{2}+u_{1, \mu}^{2}\right) \mathrm{d} x-\frac{5}{4} \mu \int_{\mathbb{R}^{3}} f u_{1, \mu} \mathrm{d} x \leqslant I^{\infty} .
$$

Using Hölder's inequality and (4.3), we see that $u_{1, \mu}$ is bounded in $H^{1}\left(\mathbb{R}^{3}\right)$ if $\mu \rightarrow 0$. Using the same method as in the proof of Theorem 3.1, we get that $u_{1, \mu}$ converges strongly in $H^{1}\left(\mathbb{R}^{3}\right)$. As $u_{1, \mu}$ is a solution of Equation (1.1), we can take the limit in Equation (1.1) when $\mu \rightarrow 0$, and obtain the result as required.

Finally, we prove Theorem 1.2.

Proof of Theorem 1.2. The function

$$
I_{\mu, f}(u)=\frac{1}{2} \int_{\mathbb{R}^{3}}\left(|\nabla u|^{2}+u^{2}\right) \mathrm{d} x-\frac{1}{4} \iint_{\mathbb{R}^{3} \times \mathbb{R}^{3}} \frac{u^{2}(x) u^{2}(y)}{|x-y|} \mathrm{d} x \mathrm{~d} y-\mu \int_{\mathbb{R}^{3}} f u \mathrm{~d} x
$$

is a continuous function of $\mu$ for any $u \in H^{1}\left(\mathbb{R}^{3}\right)$.

We assume that Equation (1.1) has at least two positive solutions when $\mu=\mu^{*}$, then deduce a contradiction. Since $I_{\mu^{*}, f}(u)$ has at least two critical points and $I_{\mu, f}(u)$ is a continuous function of $\mu$, there is a positive constant $\varepsilon_{1}>0$ such that $I_{\mu, f}(u)$ has at least two critical points for $\mu=\mu^{*}+\varepsilon_{1}$. This means that Equation (1.1) has at least two positive solutions for $\mu=\mu^{*}+\varepsilon_{1}$, which contradicts the definition of $\mu^{*}$. Hence if $\mu=\mu^{*}$, there is no more than one positive solution.

On the other hand, we can prove that $u_{0, \mu}$ and $u_{1, \mu}$ are bounded in $H^{1}\left(\mathbb{R}^{3}\right)$ for $\mu \in$ $\left(0, \mu^{*}\right)$. Using the same method as in the proof of Proposition 4.2 , we find that $u_{0, \mu}$ and $u_{1, \mu}$ converge strongly in $H^{1}\left(\mathbb{R}^{3}\right)$ as $\mu \rightarrow \mu^{*}$, and that these limits are solutions of Equation (1.1).

Combining these two results, we see that for $\mu=\mu^{*}$ there is only one positive solution for Equation (1.1). Because there are two solutions when $\mu$ is less than $\mu^{*}$, and there is no solution when $\mu$ is greater than $\mu^{* *}$, there is only one solution at $\mu^{*}$. From Rabinowitz's results (Bifurcation Theorem [15]), we know that $\mu=\mu^{*}$ is a bifurcation point of Equation (1.1).

Acknowledgements. Z.Z. was partly supported by the Volkswagen Foundation of Germany and the NSFC of China. Z.Z. thanks Professor A. Ambrosetti for a valuable suggestion, and thanks the SISSA for their support. 


\section{References}

1. A. Ambrosetti and J. L. GÁmez, Branches of positive solutions for some semi-linear Schrödinger equations, Math. Z. 224 (1997), 347-362.

2. B. Buffoni, L. JeAnjean And C. A. Stuart, Existence of a non-trivial solution to a strongly indefinite semi-linear equation, Proc. Am. Math. Soc. 119 (1993), 179-186.

3. D. M. CAO AND H. S. ZHOU, On the existence of multiple solutions of non-homogeneous elliptic equations involving critical Sobolev exponent, Z. Angew. Math. Phys. 47 (1996), 89-96.

4. I. Catto And P. L. Lions, Binding of atoms and stability of molecules in Hartree and Tomas-Fermi type theorems, Commun. PDEs 17 (1993), 1051-1110.

5. Y. B. DENG, Existence of multiple positive solutions of inhomogeneous semi-linear elliptic problems involving critical exponent, Commun. PDEs 17 (1992), 33-53.

6. Y. B. DENG, Existence of multiple positive solutions for $-\Delta u=\lambda u+u^{\frac{N+2}{N-2}}+\mu f(x)$, Acta Math. Sci. 13 (1993), 311-320.

7. Y. B. Deng And Y. LI, Existence and bifurcation of the positive solutions for a semilinear equation with critical exponent, J. Diff. Eqns 130 (1996), 179-200.

8. I. Ekeland, Non-convex minimization problems, Bull. Am. Math. Soc. 1 (1979), 443-474.

9. I. Fukuda And M. Tsutsumi, On the Yukawa-coupled Klein-Cordon Schrödinger equations in three-dimensional space, Proc. Jpn Acad. 51 (1975).

10. H. P. Heinz, T. Küpper And C. A. Stuart, Existence and bifurcation for nonlinear perturbations of periodic Schrödinger equation, J. Diff. Eqns 100 (1992), 341-354.

11. E. H. LIEB, Existence and uniqueness of the minimization solution of Choquard's nonlinear equation, Stud. Appl. Math. 57 (1977), 93-105.

12. P. L. Lions, The Choquard equation and related questions, Nonlin. Analysis 4 (1980), 1063-1073.

13. P. L. LIONS, The concentration-compactness principle in the calculus of variations in the locally compact case, I, Annls Inst. H. Poincaré Analyse Non Linéaire 1 (1984), 109-145.

14. P. L. LiOns, The concentration-compactness principle in the calculus of variations in the locally compact case, II, Annls Inst. H. Poincaré Analyse Non Linéaire 1 (1984), 223-283.

15. P. H. Rabinowitz, Some global results for nonlinear eigenvalue problems, J. Funct. Analysis 7 (1971), 441-467.

16. Z. J. ZHANG, Multiple solutions of non-homogeneous for related Choquard's equation. Acta Math. Sci. 20 (2000), 6-12. 